\title{
The stapedial reflex as a topographical marker of proximal involvement of the facial nerve in leprosy. A pilot study
}

\author{
LAURIE LEMIEUX*, TAMBI A. CHERIAN** \& \\ BRUCE RICHARD $\dagger$ \\ *Faculty of Medicine, University of Toronto, Toronto, Canada \\ **Department of Ear, Nose and Throat, Manipal College of \\ Medical Sciences, Pokhara, Nepal \\ $\dagger$ Department of Plastic Surgery, Western Regional Hospital, \\ Pokhara, Nepal
}

\section{Accepted for publication 6 August 1999}

\begin{abstract}
Summary This study aimed to determine the parameters necessary for a study of stapedial reflexes in leprosy patients to ascertain if the facial nerve is involved more proximally than the stylomastoid foramen. It involved leprosy patients with and without facial nerve involvement and non-leprosy controls. Clinical examination of the patients' ears, a tympanogram and audiogram to exclude conductive and sensorineural deafness, followed by the measurement of a stapedial reflex and the acoustic reflex threshold, were carried out. The number of absent reflexes and the acoustic reflex thresholds did not differ between the three groups of subjects. A definitive study would be logistically impossible. Suggestions are made as to more exact patient selection in order to demonstrate any stapedial reflex changes due to leprosy. The findings of this study do not suggest that facial nerve pathology extends proximally to the stylomastoid foramen, unless such proximal involvement is subclinical to the detection methods used.
\end{abstract}

\section{Introduction}

Leprosy is a disease that affects the skin, the peripheral nervous system and the upper respiratory tract. ${ }^{1,2}$ Measurable peripheral neuropathy occurs in about one-third of leprosy cases. ${ }^{3}$ Nerves most commonly involved are superficial dermal nerve twigs, cutaneous nerves, nerve trunks in the limbs and the trigeminal and facial cranial nerves. ${ }^{4}$ Classical understanding of neuropathy in leprosy holds that there are 'sites of predilection' of nerve involvement. These sites are generally cool in temperature or where nerves are subject to stress from mechanical shearing near joints or points at which swollen nerves are compressed by osseo-ligamentous tunnels. ${ }^{5-7}$ Recently, investigators have questioned whether nerve

Correspondence to: Bruce Richard, 5 Ledbury Close, Eccleston, St Helens, Merseyside, WA10 5NY, UK

Work attributed to Green Pastures Hospital and Western Regional Hospital. 
involvement is limited to these sites of predilection. ${ }^{6,8}$ Turkof et al. ${ }^{8,9}$ showed via intraoperative spinal root stimulation that lesions of the ulnar and median nerves were far more proximal than macroscopic nerve thickening suggested. In addition, the phenomenon of involuntary co-contraction of facial muscles after facial nerve palsy points to the proximal involvement of the facial nerve with mis-reinnervation. Many people with a recovered facial palsy from leprosy elevate their upper lip on gentle eye closure. ${ }^{6,10,11}$ This implies that regenerating axons of the zygomatic branch now innervate the buccal branches because the latter had degenerated back as far as the main facial nerve trunk. ${ }^{6}$ Does the leprous lesion extend even more proximally?

Facial paralysis (present in $4-10 \%$ leprosy cases ${ }^{6,10-12}$ ) is thought to be due to bacterial invasion of the extracranial branches of the facial nerve by $M$. leprae ${ }^{13}$ It is not known whether the intracranial portion of the nerve is involved prior to its emergence from the stylomastoid foramen. Were it involved at this level, an ascending infection of the facial nerve would most likely be responsible. Alternatively, as leprosy is a disease involving the upper respiratory tract and its mucosa, there is a potential conduit for the M. leprae into the middle ear through the eustachian tube.

The stapedial reflex has been described as a topographical marker to help delineate the site of a lower motor neuron facial nerve palsy. ${ }^{14}$ Absent acoustic reflexes or elevated thresholds have been reported in non-leprosy patients with unilateral facial nerve paralysis with a presumed viral aetiology, ${ }^{15}$ so-called Bell's palsy. We predicted that if there were intracranial involvement of the facial nerve in leprosy, the acoustic reflexes would be elevated or absent in leprosy patients with facial nerve palsy, whereas they would be normal in people with leprosy but no facial palsy and normal in controls.

This pilot study was undertaken to assess the feasibility of setting up a study to test this hypothesis.

\section{Materials and methods}

The nerve to the stapedius muscle is the first branch of the facial nerve after the genu and the second last branch prior to the exit of the nerve from the cranium. ${ }^{16}$ Unlike other intracranial branches of the facial nerve, its integrity is easily measured, via the multisynaptic acoustic reflex (Figure 1). This reflex is protective, occurring when the ear is exposed to loud sound. The afferent pathway is via the vestibulocochlear nerve and efferent nerve fibres are from the facial branch to the stapedius muscle. The afferent pathway synapses on both ipsilateral and contralateral nuclei in the brainstem; thus stimulation of one ear results in efferent activation in both ears. Upon stimulation, the stapedius muscle contracts, stiffening the stapedial tendon, which in turn limits the movement of the stapes and dampens the conductance of loud sounds from the middle to the inner ear. The acoustic reflex provides simple access to the proximal portion of the facial nerve. The reflex is determined by administering suprathreshold tones to the ear and measuring the change in compliance of the middle ear. For the ipsilateral reflex, the test signal and the response are measured in the same ear. A probe tip is inserted into the test ear, the right ear in the example in Figure 1. A microphone in the probe sends a series of pure tone signals at octave intervals from 250 to $4000 \mathrm{~Hz}$ into the right ear. The intensity of the signal is increased from 0 decibels hearing level ( $\mathrm{dB} \mathrm{HL}$ ) until the same probe detects a change in volume of the external auditory canal. This is usually at $85-95 \mathrm{~dB}$ HL. The change in volume reflects the contraction of the stapedius muscle as it pulls on the ossicular 


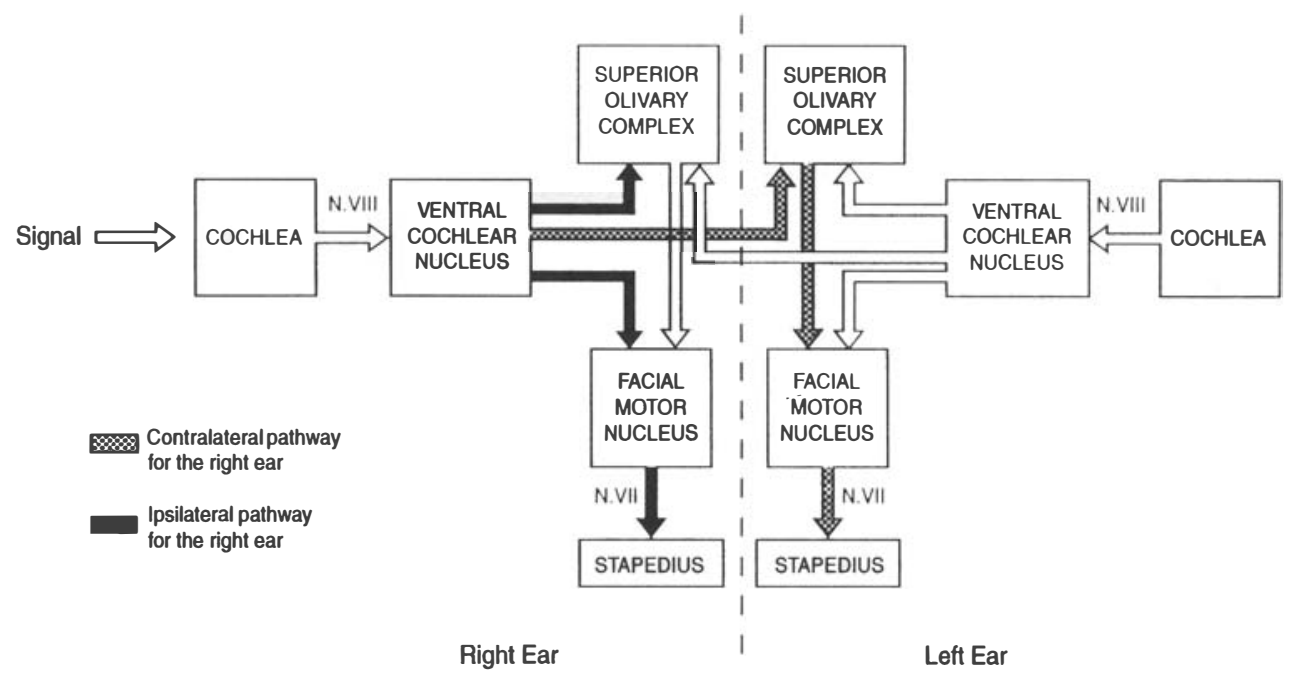

Figure 1. Schematic diagram of the neuronal pathways for ipsilateral and contralateral acoustic reflexes.

chain, displacing the tympanic membrane and thus changing the dimensions of the middle ear. The volume of the canal is a surrogate for the change in compliance of the tympanic membrane, the true reflection of the integrity of the stapedius muscle and its innervation. For the contralateral reflex of the right ear, pure tones are delivered to the right ear via a headphone and the probe tip measures the reflex contractions in the left ear. In this study ipsilateral and contralateral reflexes were taken from subjects if they had normal hearing and normal tympanograms in both ears.

Before testing the stapedial reflex as a topographical marker of a facial nerve lesion, it was important to delineate factors that could confound our results. In one study of audiovestibular status in persons affected by leprosy in India, $8 \%$ of subjects had absent or elevated reflexes, but no mention was made of facial nerve involvement or hearing impairment. ${ }^{17}$ Hearing impairment from conductive (external or middle ear), cochlear or vestibulocochlear nerve pathology can result in absent reflexes or elevated reflex thresholds. ${ }^{15}$ The prevalence of hearing impairment in the people of Nepal over 5 years of age is $17 \%{ }^{18}$ There are reports of middle ear pathology and resultant conductive hearing loss in leprosy. ${ }^{17,19}$ Cochlear deafness in leprosy has also been described. ${ }^{20}$ The prevalence of hearing loss in the leprosy population has been reported to be as high as $44-82 \% ;{ }^{17,19,20}$ this variability can be explained by differences in study sample size, selection and definition of hearing loss. Leprologists do not normally consider hearing loss to be a clinical issue; nevertheless, these studies suggest the leprosy population is more frequently hearing impaired than the normal population. We questioned whether we could obtain enough subjects from our leprosy population who had facial nerve palsy but were not hearing impaired. Reference data on normal acoustic reflex thresholds for Nepalis are unavailable. Western literature reports normal acoustic reflex thresholds between 80 and $90 \mathrm{~dB} \mathrm{HL}$ (decibel hearing level) for activator signals 250, 500, 1000, 2000 and $4000 \mathrm{~Hz} .{ }^{15}$ Elevated or abnormal thresholds are considered by some to be above $95 \mathrm{~dB}$ HL for pure tones between 250 and $2000 \mathrm{~Hz}$ and above $100 \mathrm{~dB} \mathrm{HL}$ for $4000 \mathrm{~Hz},{ }^{15,21}$ while others state that abnormal 
thresholds are those above $100 \mathrm{~dB}$ HL across all frequencies. ${ }^{15}$ These studies do not mention the racial makeup of their subject groups. Nepalis may not share the same stapedial nerve reflex profile of the people in the reported studies. We therefore conducted a study which would: 1) document the yield of subjects who had leprosy and normal hearing, 2) provide some information on normal acoustic reflex thresholds in Nepalese controls and 3) test the use of the acoustic reflex as an indicator of proximal facial nerve pathology.

\section{Subjects}

Subjects with leprosy were recruited from the inpatient population at Green Pastures Hospital, a regional leprosy treatment facility in Pokhara, Nepal. Control subjects were recruited from visitors and the support staff at the Western Regional Hospital, Pokhara.

Subjects with facial nerve palsy were selected on the basis of voluntary muscle testing (VMT) and chart review. Severity of facial nerve palsy in all five extra cranial branches of the facial nerve was documented prior to audiometric testing. All subjects underwent screening for ear pathology by the participating otolaryngologist. Controls were admitted to the study only if both ears were healthy. Leprosy subjects were admitted to the study if they had at least one healthy ear. This was because the pool of subjects for leprosy patients was more limited and yet it was possible to obtain a portion of the acoustic reflex data with one ear only. The criteria for entering only one ear to the study were as follows. If there was a unilateral hearing loss and an abnormal tympanogram in one ear, but normal hearing and a normal tympanogram in the other ear, only an ipsilateral reflex from this 'good' ear was possible. If the first ear had a hearing loss but a normal tympanogram and the other ear had normal hearing and a normal tympanogram (good ear) then ipsilateral and contralateral reflexes were taken from the 'good ear'. The contralateral reflex only requires normal hearing in the ear receiving the pure tone signal and normal middle ear and facial nerve function in the opposite ear. Pure tone audiometry was carried out on each subject and the pure tone average obtained (the average of thresholds at 500,1000 and $2000 \mathrm{~Hz}$ ).

There is no universal definition of normal hearing. Jerger ${ }^{22}$ defined normal hearing as pure tone thresholds at or below $20 \mathrm{~dB}$ HL between 500 and $8000 \mathrm{~Hz}$, while most classification systems define hearing loss as thresholds greater than $25 \mathrm{~dB}$ HL. ${ }^{23}$ Subjects were included if the tested ear had a pure tone average below $26 \mathrm{~dB}$ HL. Tympanograms and acoustic reflex thresholds were then obtained for qualif ying ears. Ears were disqualified if they had abnormal tympanograms. Ipsilateral and contralateral reflexes were obtained for pure tone signals at 250,500, 1000 and $2000 \mathrm{~Hz}$. These stimuli produce acoustic reflex thresholds that do not change with age. ${ }^{15}$

Pure tone audiometry was conducted in a sound treated room that met American National Standards Institute (ANSI) standards for acceptable ambient noise levels as measured by a Breuel and Kjaer sound level meter. ${ }^{24}$ Tympanometry and acoustic reflex testing was carried out on an Interacoustics AZ 26 Impedence Audiometer. Pure tone audiometry was conducted with an AC4 Kamplex Clinical Audiometer with Audiocups earphones. Both audiometers were certified as calibrated by an Interacoustics service technician prior to the study. The automatic mode was used for tympanometry and reflex testing, since the investigator conducting the audiometric tests could not be blinded to subject group. Increments of $1 \mathrm{~dB}$ were used for reflex testing. Reflex records were reviewed later by the otolaryngologist blinded to subject group. 


\section{Results}

Seventeen subjects with facial palsy due to leprosy were recruited, eight $(47 \%)$ of whom qualified with one or both ears normal. Of these eight subjects, 12 ears gave 12 valid ipsilateral reflex measurements and 11 valid contralateral measurement. Of those with leprosy alone, 49 people were recruited but only 17 subjects (35\%) qualified, rendering 31 ipsilateral and 29 contralateral reflex measurements. Of the 62 controls recruited, 27 (44\%) qualified with two healthy ears, that is 52 ears with 52 ipsilateral and 52 contralateral reflex measurements. All three groups were comparable in age and sex distribution (Tables 1 and 2). Seventy-one percent of the subjects were male and 29\% female. Age ranged from 16 to 57 years. The median age was 37 years.

Of the 27 subjects who had leprosy, eight had lepromatous leprosy (LL), six had borderline lepromatous leprosy (BL), nine had borderline tuberculoid leprosy (BT) and two had borderline borderline leprosy (BB). The distribution of leprosy type between the leprosy subjects with and without facial nerve involvement is shown in Table 3. Leprosy subjects had a median history of 4 years and the range was from 0.5 to 40 years.

All the acoustic reflex and threshold results were analysed by a medical statistician and subjected to parametric and non parametric testing, but Chi-square and Kruskal-Wallis values are not presented, as the study was a pilot one and the sample size was inadequate for any definitive results or trends to be valid. The median pure tone average for each group is listed in Table 4. The median acoustic reflex thresholds did not appear to differ much between the three groups. However, there was a tendency for thresholds to be slightly lower in the facial palsy group (Table 5). Variances about the mean in the leprosy groups were large. Absent reflexes occurred in all subject groups but were not significantly absent in any one group.

Table 1. Sex distribution by subject group

\begin{tabular}{lcccc}
\hline & Leprosy and facial palsy & Leprosy only & Controls & Total \\
\hline Female & 1 & 4 & 10 & 15 \\
Male & 7 & 13 & 17 & 37 \\
Totals & 8 & 17 & 27 & 52 \\
& & & & \\
\hline
\end{tabular}

Table 2. Age distribution by subject group

\begin{tabular}{lc}
\hline Leprosy and facial palsy & Median age (years) range \\
\hline Leprosy and facial palsy & $42(17-55)$ \\
Leprosy only & $35(16-57)$ \\
Controls & $35(16-57)$ \\
\hline
\end{tabular}


Table 3. Distribution of leprosy type between subject groups

\begin{tabular}{lccr}
\hline Leprosy type & Leprosy and facial palsy & Leprosy only & Total \\
\hline BT & 4 & 5 & 9 \\
BB & 1 & 1 & 2 \\
BL & 2 & 4 & 6 \\
LL & 1 & 7 & 8 \\
Total & 8 & 17 & 25 \\
\hline
\end{tabular}

\section{Discussion}

In this small pilot study, an absent reflex in leprosy patients with facial palsy was no more likely than in those with no facial nerve palsy or in those without leprosy. Furthermore, acoustic reflexes were not elevated in the facial nerve palsy group when compared to leprosy alone or to controls. Type of leprosy did not influence threshold values. While the numbers of reflexes in the groups were small and variances were large, the results were consistent across frequency and method (ipsilateral or contralateral stimulation). Even in ears rejected from the study, there was no difference in absent reflexes between the two leprosy groups and the qualifying controls, despite hearing loss. Due to the phenomenon of mis-reinnervation we presume that leprosy involves the facial nerve up to its main trunk. ${ }^{6,10,11}$ The results from this study suggest that facial nerve pathology may end distal to the stylomastoid foramen. The warmer temperature within the temporal bone may not be hospitable to M. leprae. ${ }^{25-27}$ However, in a previous study of biopsies of non-functioning facial nerve from leprosy patients with irreversible facial nerve palsies, we have established that there are large numbers of unmyelinated axons, often in regeneration clusters. This finding was evenly distributed throughout the facial nerve branches and between the fascicles. This was seen as possible evidence of proximal compression of the nerve trunk. ${ }^{28}$ If this is the case, we thought we might find absent or elevated thresholds for stapedial reflexes in such patients. Recently a dermatologist in Brazil, managing 10 leprosy patients with an acute facial patch in reversal reaction with minimal or no facial nerve paralysis, used magnetic resonance imaging (MRI) to delineate the lesions more accurately. As an incidental finding, the intratemporal portion of the facial nerve on the facial patch involved side, was noted to be irregularly thickened. This was interpreted as oedema of the nerve and the patients started on steroids to prevent facial nerve palsy (personal communication, Jane Yamashita, Brazil). This supports our hypothesis of possible compression neuropathy in the facial nerve biopsies. This oedema could be in those extracranial fascicles of the facial nerve affected by leprosy, but not involving the intratemporal branches of the facial nerve. So whilst this pilot study did

Table 4. Median pure tone averages for each subject group ( $n=$ sample size of ears)

\begin{tabular}{lccc}
\hline & Leprosy and facial palsy & Leprosy only & Controls \\
\hline Pure tone average, dB HL & $(n=12)$ & $(n=31)$ & $(n=52)$ \\
\hline
\end{tabular}


Table 5. Median acoustic reflex thresholds for each stimulus frequency across subject groups $(n=$ sample size of ears)

\begin{tabular}{|c|c|c|c|c|c|c|}
\hline $\mathrm{Hz}$ & Ipsi 500 & Ipsi 1000 & Ipsi 2000 & Contra 500 & Contra 1000 & Contra 2000 \\
\hline Leprosy and facial palsy & $\begin{array}{l}98 \cdot 0 \\
(n=12)\end{array}$ & $\begin{array}{c}98 \cdot 0 \\
(n=12)\end{array}$ & $\begin{array}{l}109 \cdot 5 \\
(n=12)\end{array}$ & $\begin{array}{l}101 \cdot 0 \\
(n=11)\end{array}$ & $\begin{array}{l}94 \cdot 0 \\
(n=11)\end{array}$ & $\begin{array}{c}97.0 \\
(n=11)\end{array}$ \\
\hline Leprosy only & $\begin{array}{l}102 \cdot 0 \\
(n=31)\end{array}$ & $\begin{array}{l}100 \cdot 5 \\
(n=30)\end{array}$ & $\begin{array}{l}110 \cdot 0 \\
(n=29)\end{array}$ & $\begin{array}{l}102 \cdot 5 \\
(n=30)\end{array}$ & $\begin{array}{l}97 \cdot 0 \\
(n=30)\end{array}$ & $\begin{array}{l}97.5 \\
(n=30)\end{array}$ \\
\hline Controls & $\begin{array}{l}103 \cdot 0 \\
(n=50)\end{array}$ & $\begin{array}{l}102 \cdot 0 \\
(n=52)\end{array}$ & $\begin{array}{l}110 \cdot 0 \\
(n=51)\end{array}$ & $\begin{array}{l}102 \cdot 0 \\
(n=51)\end{array}$ & $\begin{array}{l}99.0 \\
(n=51)\end{array}$ & $\begin{array}{l}100 \cdot 0 \\
(n=49)\end{array}$ \\
\hline
\end{tabular}

not indicate a consistent intratemporal specific facial nerve deficit, it remains extremely likely that leprous neuropathology could cause an intratemporal compression neuropathy and thus be implicated in the more severe types of total facial nerve palsy.

Less than half the facial palsy subjects qualified for this study on the basis of abnormal external and middle ear anatomy, abnormal tympanometry or abnormal hearing. Our results are consistent with the $44 \%$ prevalence rate of hearing loss in persons affected by leprosy found by Mann et al. ${ }^{20}$ Of the few studies of cochleo-vestibular function in leprosy patients some demonstrate a mild sensorineural hearing loss (of the order of 16-60 dB hearing loss) in $44-60 \%$ of lepromatous patients. ${ }^{17,20}$ One recent small study showed no hearing loss in 19 lepromatous patients. ${ }^{29}$ The former studies also suggest that it is the cochlear portion of the eighth nerve that is involved and not the vestibular apparatus.

Two recent studies of auditory brain stem evoked potentials in leprosy patients suggest a lesion between the cochlear and the lateral lemniscus in the auditory brain stem pathway. ${ }^{29,30}$ We noted a difference of $10 \mathrm{~dB}$ between the ipsilateral and contralateral stapedial reflex thresholds at $2000 \mathrm{~Hz}$. The difference, however, was that the ipsilateral threshold was $10 \mathrm{~dB}$ higher than the contralateral, whilst the expected finding would be no difference or a small $(2-5 \mathrm{~dB})$ higher contralateral threshold. ${ }^{15}$ Again, the variance is large, so this may not be the correct interpretation. This finding provides no evidence to refute or support a lower midbrain leprous pathology.

Controls were excluded for abnormal tympanic membranes, abnormal tympanometry or hearing loss. The rejection rate for our controls was $56 \%$. This is over 3 times the reported national average for hearing impairment. ${ }^{18} \mathrm{We}$ believe one reason for this was that when people who thought they might have a hearing problem found out about our study, they volunteered so that they could have quick and free access to testing.

While this was not a normative study on acoustic reflex thresholds in Nepalese people, the thresholds obtained were, in most cases, above normal values reported in the literature. ${ }^{15,31}$ This may be related to the automatic testing mode used, as the stimulus tone may have been rising while the reflex was initiated and thus the reflex was given at a higher threshold than may have been recorded manually. Alternatively, it may reflect a racial or environmental effect of life in Nepal.

Testing of the stapedial branch of the facial nerve in leprosy has not been previously reported. The acoustic reflex test is a simple, non-invasive method for accessing this intratemporal branch of the facial nerve. The greater petrosal and chorda tympani branches, whilst amenable to quantifiable testing (Schirmer test, salivary flow test and electrogustometry), ${ }^{1}$ 
fail to differentiate between an intratemporal or an end organ aetiology for any abnormal findings in the case of leprosy. None of our patients reported aguesia, nor did we attempt to test for any taste loss. We have previously, seen two patients with catastrophic facial nerve palsy reporting diminished taste sensibility.

Little clinical attention is paid to hearing loss in leprosy, as it is presumed that leprosy does not affect cranial nerves intracranially. ${ }^{20}$ Our results show mild deafness to be a problem in approximately $50 \%$ of persons affected by leprosy, whilst the prevalence of hearing impairment in the people of Nepal over 5 years of age is $17 \% .{ }^{18}$ We suggest that hearing assessment should be a standard component of intake assessment at our leprosy treatment centre.

In view of the small differences between the reflex thresholds in the three groups and the large variance of thresholds between patients, a definitive study would need to be very large. Given that no consistent large differences were found in this pilot study, the clinical value of a large study would also be unsure. We therefore suggest evaluating the acoustic reflex technique as a research tool in the following two situations.

- Test subjects with a trigeminal patch in reaction, or a recent new facial nerve palsy to determine whether there is involvement of the intratemporal facial nerve during this acute phase. This would be corroborative evidence of intratemporal compression.

- Test subjects prior to steroid treatment for reversal reactions resulting in facial palsy and compare the thresholds with those obtained after successful treatment.

Routine otological and audiometric testing would provide leprologists with further information about nerve involvement beyond the stylomastoid foramen and surgeons information with which to avoid incomplete neurolysis.

\section{Acknowledgements}

The authors wish to acknowledge the logistical support of Binod Koirala and Yam Kumar Pun, the audiological advice of Bradley Stephenson and the statistical expertise of Dr Alison Anderson, without which this project would not have been possible. Laurie Lemieux thanks Dr J Keystone and the University of Toronto Medical Alumni Association for the International Health Scholarship which allowed her to participate in this study.

\section{References}

${ }^{1}$ Rathi SS, Chaturvedi VN, Raizada RM, Jain SKT. Electrogustometry in Hansen's disease (study of 225 cases). Int $J$ Lepr, 1986; 54: 252-255.

2 van Brakel WH. Peripheral neuropathy in leprosy: the continuing challenge. Thesis/Dissertation, Utrecht University, 1994; 1-217.

3 van Brakel WH, Khawas IB. Nerve damage in leprosy: a n epidemiological study of 396 patients in West Nepalpart 1:definitions, methods and frequencies. Lepr Rev, 1994; 65: 204-221.

4 Srinivasan H. Disability, deformity and rehabilitation. In: Hastings RC, ed. Leprosy. Edinburgh: Churchill Livingstone, 1994; 411-447.

5 Ridley DS. Neuropathy in leprosy. In: Pathogenesis of leprosy and related diseases. London: Butterworth \& Co. Ltd, 1988; 71-83.

${ }^{6}$ Richard BM, Corry PR. The cervical branch of the facial nerve in leprosy. Int J Lepr, 1997; 65: 170-177.

7 Dastur DK. Pathology and pathogenesis of predilective sites of nerve damage in leprous neuritis. Neurosurg Rev, 1983; 6: 139-152. 
${ }^{8}$ Turkof E, Tambwekar S, Mansukhani K, Millesi H, Mayr N. Intraoperative electroneurodiagnostics to detect a second granuloma in the cubital area of the median nerves affected by leprosy: a new approach to prevent incomplete surgery. Int J Lepr, 1995; 63: 409-416.

9 Turkof E, Tambwekar S, Mansukhani K, Millesi H, Mayr N. Intraoperative spinal root stimulation to detect most proximal site of leprous ulnar neuritis. Lancet 1994; 343: 1604-1605.

${ }^{10}$ Lubbers WJ, Schipper A, Hogeweg M, Soldenhoff DR. Paralysis of facial muscles in leprosy patients with lagophthalmos. Int J Lepr, 1994; 62: 220-224.

11 Ranney DA. The prevalence and consequences of mis-reinnervation in facial neuritis. Int J Lepr, 1974; 42: 316-322.

12 Antia NH, Divekar SC, Dastur DK. The facial nerve in leprosy I. Clinical and operative aspects. Int J Lepr, 1966; 34: 103-117.

13 Job CK. Pathology of leprosy. In: Hastings RC, ed. Leprosy. Edinburgh: Churchill Livingstone, 1994; $193-224$.

14 Wiley TL, Fowler CG. Acoustic immittance measures in clinical audiology. San Diego: Singular Publishing Group, 1997; 100-101.

15 Wilson RH, Margolis RH. Acoustic-reflex measurements. In: Rintelmann WF, ed. Hearing assessment. Needham Heights: Allyn and Bacon, 1991; 247-319.

16 Last RJ. Anatomy, regional and applied. Singapore: Longman Singapore Publishers Pte. Ltd, 1986; 551-552.

17 Singh T, Agrawal SK, Bajaj AK, Singh RK, Singh MM. Evaluation of audiovestibular status in leprosy. Ind J Lepr, 1984; 56: 24-29.

18 Little P, Bridges A, Guragain RPS, Thapa SR, Raut SP. Survey of the prevalence of deafness and ear disease in Nepal. Kathmandu, Nepal: Otology Programmed Development Committee, Tribuvan University. Echoes, 1998; 12-91.

19 Srinivasan KPI. Hearing problems among leprosy sufferers. New Delhi, XII Int Lepr Congress, 1984; $21-24$.

20 Mann SBS, Kumar B, Yande R, Kaur S, Kaur I, Mehra YN. Eighth nerve evaluation in leprosy. Ind JLepr., 1987; 59: $20-25$.

${ }^{21}$ Hayes DH, Jerger J. Impedence audiometry in otologic diagnosis. Otolaryngol. Clin. N. Am., 1978; 11 : 765.

22 Jerger J, Jerger S. Measurement of hearing in adults. In: Paparella MM, Shumrick DA, eds. Otolaryngology. Philadelphia: WB Saunders, 1980; 1226.

23 Clark JG. Uses and abuses of hearing loss classification. American Speech and Hearing Association's Magazine, 1981; 493-500.

24 Brown S. ANSI S3.1-1991 (ASA99). In: Kerr AG, ed. Scott-Brown's otolaryngology. Oxford: ButterworthHeinemann, 1997.

25 Enna CD, Berghtholdt HT, Stockwell F. A Study of surface and deep temperatures along the course of the ulnar nerve in the pisohamate tunnel. Int J Lepr, 1974; 42: 43-47.

26 Sabin TD. Temperature-linked sensory loss: a unique pattern in leprosy. Arch Neurol, 1969; 20: $257-262$.

27 Sabin TD, Hackett ER, Brand PW. Temperatures along the course of certain nerves often affected in lepromatous leprosy. Int J Lepr, 1974; 42: 38-42.

28 Richard BM and Jacobs JM. Facial nerve pathology in leprosy: searching for the proximal extent of the lesion in facial nerve biopsies. Lepr Rev, 1999; 70: 000-000.

29 Celik O, Yalcin S, Gok U, Yavrucuoglu E, Ozturk A, Akyol A. Auditory brain stem evoked potentials in patients with leprosy. Int J Lepr, 1997; 65: 166-169.

30 Kochar DK, Gupta DV, Chauhan S, Halwai M, Kumawat BL. Study of brain stem audutory-evoked potentials (BAEPs) and visual-evoked potentials (VEPs) in leprosy. Int J Lepr, 1997; 65: 157-165.

31 Jerger J, Oliver TA, Jenkins H. Suprathresholds abnormalities of the stapedius reflex in acoustic tumor. Ear and Hearing, 1987; 8: 131-139. 